\title{
A self-consistent Hirshfeld method for the atom in the molecule based on minimization of information loss
}

Dieter Ghillemijn ${ }^{1,2}$, Patrick Bultinck ${ }^{1,2, *}$, Dimitri Van Neck ${ }^{2,3}$ and Paul W. Ayers ${ }^{4}$

1. Department of Inorganic and Physical Chemistry, Ghent University

Krijgslaan 281 (S3), 9000 Gent, Belgium

2. Members of the Ghent-Brussels Quantum Chemistry and Molecular Modeling alliance

3. Center for Molecular Modeling, Ghent University

Technologiepark 903, 9052 Zwijnaarde, Belgium

4. Department of Chemistry, McMaster University

Hamilton, Ontario L8S 4M1, Canada 


\begin{abstract}
Based on the so-called Hirshfeld atom in the molecule (AIM) scheme, a new AIM method is presented. The method is similar to the Hirshfeld-I scheme, with the AIM weight function being constructed by minimizing the information loss upon formation of the molecule, but now requiring explicitly that the promolecular densities integrate to the same number of electrons as the AIM densities. This new weight function leads to a new iterative AIM scheme, and the resulting operative scheme is examined and discussed. The final results indicate that the newly proposed method does not perform as well as the Hirshfeld-I method.
\end{abstract}

\title{
1 Introduction
}

Most of our understanding of chemistry is based on regarding molecules as composed of atoms held together by chemical bonds. Despite the tremendous amount of scientific work that has been done starting from this view, no definite theoretical framework has been sketched for the chemical bond and atoms in molecules (AIM). In this paper we focus on the definition of the atom in the molecule and introduce a new AIM technique.

Many different AIM methods have been published, varying from methods based on the attachment of basis functions to atomic centers, as in the Mulliken $[1,2,3,4]$ approach, to techniques where a three-dimensional characterization of the AIM is introduced. For the latter, the AIM density function $\rho_{A}(\vec{r})$ can in general be defined as [5]:

$$
\rho_{A}(\vec{r})=w_{A}(\vec{r}) \rho(\vec{r}),
$$

with $\rho(\vec{r})$ the molecular electron density. The factor $w_{A}(\vec{r})$ is a positive semidefinite weight function fulfilling

$$
\forall \vec{r}: \sum_{A} w_{A}(\vec{r})=1
$$

There is no unique choice for the weight function. The two main lines of thought are characterized by either a binary division of 3D space among the atoms, or a more fuzzy division. In Bader's Quantum Chemical Topology (QCT) $[6,7]$, a binary approach is used where at each point in space the entire molecular electron density is ascribed to one single atom, using the zero-flux surfaces to divide the molecular electron density. This leads to sharp boundaries between the different AIM. In so-called fuzzy atom approaches, these boundaries are less strict and the electron density of every atom extends to infinity, although the AIM density function is usually still very localized.

One of the best known fuzzy atoms is the Hirshfeld [8] atom in the molecule. In that case, the weight function of an atom $\mathrm{A}$ is computed as the share of the corresponding isolated atomic density with respect to the so-called promolecular density $\rho^{0}(\vec{r})=\sum_{A} \rho_{A}^{0}(\vec{r})$ :

$$
w_{A}^{H}(\vec{r})=\frac{\rho_{A}^{0}(\vec{r})}{\sum_{B} \rho_{B}^{0}(\vec{r})} .
$$

A promolecular density is obtained as the summation of the atomic densities of the isolated atoms $\rho_{A}^{0}(\vec{r})$, centered at the same positions as the atomic nuclei in 
the real molecule. Although this method is quite popular, especially within the broad field of so-called conceptual or chemical DFT, it has been criticized on different occasions mainly because of the arbitrary choice of the isolated atomic densities $[9,10,11,12]$. As an example, the AIM density functions on Li and F in $\mathrm{LiF}$ depend quite strongly on whether the promolecule is considered to be composed of $\mathrm{Li}^{+}$and $\mathrm{F}^{-}, \mathrm{Li}^{0}$ and $\mathrm{F}^{0}$ or $\mathrm{Li}^{-}$and $\mathrm{F}^{+}$.

\section{Iterative Hirshfeld methods}

This dependence on the arbitrary choice of the atomic states used in computing the weight function is clearly problematic. Recently, some of us solved this problem via the so-called self consistent Hirshfeld-I scheme $[9,13]$ although the recent revival in the interest in Hirshfeld techniques also led to the so-called Iterated Stockholder Atoms (ISA) $[14,15,16]$. In the Hirshfeld-I procedure, which forms the basis for the present work, an iterative scheme is developed in the following way. First the classical Hirshfeld method is used starting from a set of arbitrarily chosen charge states of the atoms that compose the molecule. In practice usually a set of neutral atoms is chosen. The Hirshfeld AIM weight function is used to obtain AIM density functions according to eq. 1 and the AIM electronic populations are computed from these density functions. A new density function is computed for the AIM in its isolated state with this specific number of electrons $[17,18,19]$ :

$$
\begin{aligned}
\rho_{A}^{0}\left(\vec{r}, N_{A}\right) & =\rho_{A}^{0}\left(\vec{r}, \operatorname{lint}\left(N_{A}\right)\right)\left[\operatorname{uint}\left(N_{A}\right)-N_{A}\right] \\
& +\rho_{A}^{0}\left(\vec{r}, \operatorname{uint}\left(N_{A}\right)\right)\left[N_{A}-\operatorname{lint}\left(N_{A}\right)\right],
\end{aligned}
$$

where $\operatorname{lint}\left(N_{A}\right)$ means the integer part of $N_{A}$ and uint $\left(N_{A}\right)$ the higher integer number of $N_{A}$, or uint $\left(N_{A}\right)=\operatorname{lint}\left(N_{A}\right)+1$. This approximation is exact for exact calculations (full $\mathrm{CI}$ ), and a good approach in the computationally economic methods commonly used in conceptual DFT [20,21,22]. This interpolation uses the electron densities of the atoms at integer populations, which are available through atomic calculations. The resulting set of density functions for (often) fractional electron populations is used to compute new Hirshfeld weights. This process is repeated until self-consistency is reached, i.e. until the AIM electronic populations no longer change. It has been shown that the outcome of the procedure is independent of the choice of the set of starting AIM and that most other problems typically associated with the classical Hirshfeld method are solved $[9,23]$. Moreover, it leads to very good atomic-monopole-based molecular electrostatic potentials [24].

According to Parr and Nalewajksi $[25,26,27,28,29]$, the Hirshfeld method is deeply rooted in information theory [30]. Using the Kullback-Leibler [31] formula as a measure of missing information, the total missing information can be written as a sum of the missing information of each atom:

$$
I=\sum_{A} \int \rho_{A}(\vec{r}) \ln \left(\frac{\rho_{A}(\vec{r})}{\rho_{A}^{0}(\vec{r})}\right) \mathrm{d} \vec{r},
$$

under the requirement that

$$
\forall \vec{r}: \sum_{A} \rho_{A}(\vec{r})=\rho(\vec{r}) .
$$


The constrained minimization of Eq. 5 straightforwardly results in the Hirshfeld weight function $[25,29]$. This means that the Hirshfeld-type AIM are the AIM that minimize the information lost relative to the separated-atom limit [9]. However, there is an extra requirement that was lacking in the original Hirshfeld scheme, namely the requirement that the AIM electronic population $N_{A}$ should be the same as that of the isolated atom, $N_{A}^{0}$ :

$$
\forall A: N_{A}=\int \rho_{A}(\vec{r}) \mathrm{d} \vec{r}=\int \rho_{A}^{0}(\vec{r}) \mathrm{d} \vec{r}=N_{A}^{0} .
$$

The Hirshfeld-I scheme fulfills this constraint after convergence [9], although prior to convergence the information theory connection is blurred by the fact that the constraint is not fulfilled.

In the present paper we examine a new, strictly variational, scheme where from the start an extra constraint for each atom is imposed, namely the constraint of Eq. 7. This new AIM method is based on using a weight function that explicitly obeys the constraints of both Eq. 6 and 7. This weight function defines AIM that minimize the information lost on formation of the molecule out of its isolated atoms. The main difference between the Hirshfeld-I method and the new method lies in the organization of the different minimization steps. In Hirshfeld-I, the population constraint (Eq. 7) is imposed as an additional self-consistency condition, but remains out of the minimization procedure:

$$
\underbrace{\min }_{\left\{\left\{\rho_{A}(\vec{r})\right\} \mid \rho(\vec{r})=\sum_{A} \rho_{A}(\vec{r})\right\}} \sum_{A} \int \rho_{A}(\vec{r}) \ln \left(\frac{\rho_{A}(\vec{r})}{\rho_{A}^{0}\left(\vec{r}, N_{A}^{0}\right)}\right) \mathrm{d} \vec{r},
$$

whereas in the presently described algorithm, both constraints are placed in one minimization:

$$
\underbrace{\min }_{\left\{\begin{array}{c|c}
\left\{\rho_{A}(\vec{r})\right\} & \rho(\vec{r})=\sum_{A} \rho_{A}(\vec{r}) \\
\left\{N_{A}^{0}\right\} & \left\{N_{A}^{0}=\int \rho_{A}(\vec{r}) \mathrm{d} \vec{r}\right\}
\end{array}\right\}} \sum_{A} \int \rho_{A}(\vec{r}) \ln \left(\frac{\rho_{A}(\vec{r})}{\rho_{A}^{0}\left(\vec{r}, N_{A}^{0}\right)}\right) \mathrm{d} \vec{r} .
$$

The two algorithms do not lead to the same end result, as will be shown, and the main aim of this paper is to implement and test the new scheme, investigate its performance and compare the results with those from other Hirshfeld-type methods.

\section{The population constrained Hirshfeld method}

\subsection{Weight function}

To find the weight function that obeys both conditions, Eq. 5 has to be minimized with Eqs. 6 and 7 as linear constraints. This leads to the following Lagrangian:

$$
\begin{array}{r}
\sum_{B}\left[\int \rho_{B}(\vec{r}) \ln \left(\frac{\rho_{B}(\vec{r})}{\rho_{B}^{0}\left(\vec{r}, N_{B}^{0}\right)}\right) \mathrm{d} \vec{r}-\lambda_{B}\left(N_{B}^{0}-\int \rho_{B}(\vec{r}) \mathrm{d} \vec{r}\right)\right] \\
-\int \mathrm{d} \vec{r} \Lambda(\vec{r})\left(\sum_{B} \rho_{B}(\vec{r})-\rho(\vec{r})\right) .
\end{array}
$$


In the last part of Eq. 10, the integral and the dependence of the Lagrange multiplier $\Lambda$ on $\vec{r}$ appear because Eq. 6 must hold for every point $\vec{r}$ in space. The second part reflects the adherence to Eq. 7 (for every atom $B$ ). To find the minimal information loss, Eq. 10 must be minimized with respect to two variables: $N_{A}^{0}$ and $\rho_{A}(\vec{r})$.

The derivative of Eq. 10 with respect to $\rho_{A}(\vec{r})$ results in

$$
\ln \left(\frac{\rho_{A}(\vec{r})}{\rho_{A}^{0}\left(\vec{r}, N_{A}^{0}\right)}\right)+1+\lambda_{A}-\Lambda(\vec{r})=0 .
$$

This derivation can be done for every atom $A$. By combining Eq. 11 for an atom $A$ and an atom $B, \Lambda(\vec{r})$ can be eliminated:

$$
\ln \left(\frac{\rho_{A}(\vec{r})}{\rho_{A}^{0}\left(\vec{r}, N_{A}^{0}\right)}\right)+\lambda_{A}=\ln \left(\frac{\rho_{B}(\vec{r})}{\rho_{B}^{0}\left(\vec{r}, N_{B}^{0}\right)}\right)+\lambda_{B} .
$$

After rearrangement and summation over all atoms $B$, the following formula is obtained:

$$
\sum_{B} \rho_{B}^{0}\left(\vec{r}, N_{B}^{0}\right) e^{-\lambda_{B}}=\frac{\rho_{A}^{0}\left(\vec{r}, N_{A}^{0}\right)}{\rho_{A}(\vec{r})} \rho(\vec{r}) e^{-\lambda_{A}},
$$

using Eq. 6. A final rearrangement results in the familiar-looking formula

$$
\rho_{A}(\vec{r})=\frac{\rho_{A}^{0}\left(\vec{r}, N_{A}^{0}\right) e^{-\lambda_{A}}}{\sum_{B} \rho_{B}^{0}\left(\vec{r}, N_{B}^{0}\right) e^{-\lambda_{B}}} \rho(\vec{r}) .
$$

The derivative of Eq. 10 with respect to $N_{A}^{0}$ equals

$$
-\int \mathrm{d} \vec{r} \frac{\rho_{A}(\vec{r})}{\rho_{A}^{0}\left(\vec{r}, N_{A}^{0}\right)} \frac{\partial\left(\rho_{A}^{0}\left(\vec{r}, N_{A}^{0}\right)\right)}{\partial N_{A}^{0}}-\lambda_{A}=0,
$$

which can be rewritten as

$$
\lambda_{A}=-\int \mathrm{d} \vec{r} \rho_{A}(\vec{r}) \frac{\partial\left(\rho_{A}^{0}\left(\vec{r}, N_{A}^{0}\right)\right)}{\partial N_{A}^{0}} \frac{1}{\rho_{A}^{0}\left(\vec{r}, N_{A}^{0}\right)} .
$$

In Eq. 16 there is still a derivative left, known as the Fukui function (under the constraint of a constant external potential) $[17,20,21,32,33]$. The Fukui function in a finite difference approximation can be written as [20]

$$
\frac{\partial}{\partial N_{A}^{0}} \rho_{A}^{0}\left(\vec{r}, N_{A}^{0}\right)=\Delta \rho_{A}^{0}\left(\vec{r}, N_{A}^{0}\right)=\rho_{A}^{0}\left(\vec{r}, \operatorname{uint}\left(N_{A}^{0}\right)\right)-\rho_{A}^{0}\left(\vec{r}, \operatorname{lint}\left(N_{A}^{0}\right)\right),
$$

This approximation is exact for exact calculations [20,21,22].

\subsection{Computational algorithm}

Using the formulas derived in the previous Section, it is now possible to propose a new definition of an atom in a molecule. The new AIM method is called Hirshfeld-I $\lambda$, as it is still an iterative Hirshfeld method but now with an extra set of variables. Eqs. 14 and 16 are the two formulae that define the new AIM. 
The fraction in Eq. 14 resembles the right hand side of Eq. 3. This fraction is the new weight function that defines the AIM:

$$
w_{A}^{H I \lambda}(\vec{r})=\frac{\rho_{A}^{0}\left(\vec{r}, N_{A}^{0}\right) e^{-\lambda_{A}}}{\sum_{B} \rho_{B}^{0}\left(\vec{r}, N_{B}^{0}\right) e^{-\lambda_{B}}} .
$$

Compared to the weight function of the Hirshfeld and Hirshfeld-I scheme, this new weight function has an extra factor (both in the numerator and in the denominator) of $e^{-\lambda_{A}}$.

Eq. 16 shows us that the set $\{\lambda\}$ depends on the AIM electron densities. And as $\rho_{A}(\vec{r})=w_{A}^{H I \lambda}(\vec{r}) \rho(\vec{r})$ and $w_{A}^{H I \lambda}(\vec{r})$ depend on $\lambda_{A}$, the AIM densities depend on the set $\{\lambda\}$. This means that the two sets of parameters are interdependent; and as a result, these equations can not be solved in one calculation. Again the solution will have to be found in an iterative way. The scheme constructed bears many similarities with the Hirshfeld-I [9] scheme. The main difference is the calculation of the extra set of variables $\{\lambda\}$ and their use in the weight function. Self-consistency in the set $\{\lambda\}$ is also required to conclude convergence.

The Hirshfeld-I $\lambda$ weight function in the i'th iteration can now be written as

$$
w_{A}^{H I \lambda, i}(\vec{r})=\frac{\rho_{A}^{0}\left(\vec{r}, N_{A}^{i-1}\right) e^{-\lambda_{A}^{i-1}}}{\sum_{B} \rho_{B}^{0}\left(\vec{r}, N_{B}^{i-1}\right) e^{-\lambda_{B}^{i-1}}},
$$

with

$$
\lambda_{A}^{i}=-\int \mathrm{d} \vec{r} \rho_{A}^{i}(\vec{r}) \frac{\partial\left(\rho_{A}^{0}\left(\vec{r}, N_{A}^{i-1}\right)\right) / \partial N_{A}^{i-1}}{\rho_{A}^{0}\left(\vec{r}, N_{A}^{i-1}\right)} .
$$

As the promolecular populations are usually fractional numbers, the interpolation expression of Eq. 4 is again used.

\subsection{Convergence}

This Hirshfeld-I $\lambda$ scheme was implemented and tested, but did not converge for most of the molecules in the test set [34]. The $\lambda$ 's and the populations kept oscillating, especially when larger basis sets were used. The explanation for this oscillation was traced back to the expression for the $\lambda$ 's, Eq. 16 .

More specifically, the Fukui function causes the oscillation. Because of the discontinuity in the Fukui function, an infinitesimally small change in $N_{A}^{i-1}$ can induce a large change in $\lambda_{A}^{i}$. And as $\lambda_{A}^{i}$ is essential to compute $N_{A}^{i+1}$, this may cause a big change in $N_{A}^{i+1}$. When $N_{A}^{i+1}$ crosses an integer, the discontinuity in the Fukui function can cause these oscillations, hindering convergence or leading to unrealistic numbers.

In order to soften this discontinuity, the Fukui function is replaced by a function that softens the discontinuity, but contains a parameter $k$ to gradually reintroduce the discontinuity:

$$
f\left(N_{A}^{0}\right)=f_{l}\left(N_{A}^{0}\right)+\frac{f_{u}\left(N_{A}^{0}\right)-f_{l}\left(N_{A}^{0}\right)}{1+e^{-k\left(N_{A}^{0}-\operatorname{nint}\left(N_{A}^{0}\right)\right)}} \approx \frac{\partial}{\partial N_{A}^{0}} \rho_{A}^{0}\left(\vec{r}, N_{A}^{0}\right),
$$

with

$$
\begin{aligned}
f_{l}\left(N_{A}^{0}\right) & =\rho_{A}^{0}\left(\operatorname{nint}\left(N_{A}^{0}\right)\right)-\rho_{A}^{0}\left(\operatorname{nint}\left(N_{A}^{0}\right)-1\right), \\
f_{u}\left(N_{A}^{0}\right) & =\rho_{A}^{0}\left(\operatorname{nint}\left(N_{A}^{0}\right)+1\right)-\rho_{A}^{0}\left(\operatorname{nint}\left(N_{A}^{0}\right)\right),
\end{aligned}
$$


and $\operatorname{nint}\left(N_{A}^{0}\right)$ meaning the integer number nearest to $N_{A}^{0}$. This formula creates a step function around the nearest integer number, in the interval $\left[\operatorname{nint}\left(N_{A}^{0}\right)-\right.$ $\left.0.5 ; \operatorname{nint}\left(N_{A}^{0}\right)+0.5\right]$. The value of $f$ at the discontinuity (the nearest integer number) is always $\frac{1}{2}\left(f_{u}\left(N_{A}^{0}\right)+f_{l}\left(N_{A}^{0}\right)\right)$.

In Eq. 21 the parameter $k$ (also called the order of the function) defines the sharpness of the step. A larger $k$ makes the function sharper, and for $k \rightarrow \infty$ the original integer discontinuity in the Fukui function is regained. This means that a larger $k$ results in a better approximation of the Fukui function, but it also increases the risk of not reaching convergence. Therefore, an extra iterative scheme is introduced in the following way:

1. Choose starting values for the set $\{\lambda\}$, and set the promolecular populations equal to the nuclear charges. (Setting all $\lambda$ 's to zero is a good choice, this way the first calculation is a classical Hirshfeld analysis.) Choose a (low) starting value for $k$.

2. Perform the Hirshfeld-I $\lambda$ scheme, using this value of $k$.

3. Raise $k$ with a specific amount.

4. Perform the Hirshfeld-I $\lambda$ scheme, using the latest value of $k$. Initialize the sets $\{\lambda\}$ and $\{N\}$ with the converged values at the previous value of $k$.

5. Assess the differences in $\{\lambda\}$ and $\{N\}$ between two subsequent values of $k$. If convergence was not yet obtained, return to step 3 .

This Hirshfeld-I $\lambda$ scheme comprises two iterative loops, an inner loop (seeking convergence at a certain $k$ ) and an outer loop (seeking convergence at different $k$ 's). The scheme stops when the $\lambda$ 's and populations at different values of $k$ are converged (outer loop), or when the calculations at a certain $k$ no longer converge (inner loop).

Two remarks should be made concerning this scheme. First, it is important to start the calculation at a certain value of $k$ with the converged populations of the previous $k$ as promolecular populations. The inner iteration converges much faster this way. It is important to keep the calculation time in mind, as the two nested loops form a laborious scheme in terms of computational work. The second remark is about the starting value of $k$. If the starting value is taken too low, the step function introduces the discontinuities in other places, instead of softening them. This must of course be avoided. Starting at too high values of $k$ is not good either, as the chance of not reaching convergence in the first loop rises with increasing $k$.

The scheme discussed in the previous Section was implemented. The results showed that the calculations ended before the populations had converged as a function of $k$. To make sure that the inner loops can still find convergence at relatively high values of $k$, the changes in populations and $\lambda$ 's are damped. This quenches the oscillations in the inner loops, which allows the outer loop to reach convergence. This means that after every calculation of $N_{A}$ 's or $\lambda_{A}$ 's, the difference with the previous values is quenched using the formula

$$
y_{q}^{i}=y^{i-1}+q\left(y^{i}-y^{i-1}\right),
$$




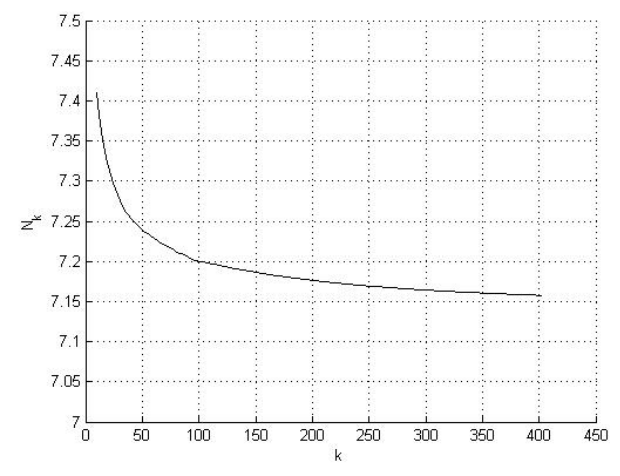

Figure 1: Hirshfeld-I $\lambda$ population as a function of the order $k$ of the fitting function (for the $\mathrm{N}$ atom of $\mathrm{CH}_{3} \mathrm{NH}_{2}$ at $\mathrm{HF} / 6-311++\mathrm{G}^{* *}$ level of theory).

with

$$
0 \leq q \leq 1
$$

and $y$ can be $N_{A}$ or $\lambda_{A} \cdot y_{q}^{i}$ corresponds to the value of $y$ to be used in the next iteration instead of the $y^{i}$ that would be used when not using this quenching formula. This $q$ is called the quench factor, and a quench factor of 0.1 was found optimal. Figure 1 shows an example of the convergence of a Hirshfeld-I $\lambda$ population as a function of $k$. The scheme clearly converges. In this example, at $k$ equal to 200 all populations and $\lambda$ 's differed less than 0.0001 compared to the values at the previous $k(k=198)$.

\subsection{Uniqueness of the solution}

In [16] the solution to the ISA scheme was shown to be variationally derivable as the mininum of an underlying functional, and uniqueness of the minimum was established by proving the convexity of the functional. As mentioned in Section 2 , the Hirshfeld-I method is not completely variational, though the resulting self-consistency scheme was checked to have a unique solution by extensive testing $[9,23]$, starting from different initial charge states of the promolecular atoms. The presently proposed Hirshfeld-I $\lambda$ scheme is strictly variational, so it is instructive to investigate the possible convexity of the underlying functional and thus the uniqueness of the calculated Hirshfeld-I $\lambda$ charges.

To simplify the mathematics, we rephrase the variational problem (Eq. 9) by eliminating the $N_{A}^{0}$ variables, keeping only the $\rho_{A}(\vec{r})$ degrees of freedom:

$$
\underbrace{\min }_{\left\{\left\{\rho_{A}(\vec{r})\right\} \mid \rho(\vec{r})=\sum_{A} \rho_{A}(\vec{r})\right\}} \sum_{A} \int \rho_{A}(\vec{r}) \ln \left(\frac{\rho_{A}(\vec{r})}{\rho_{A}^{0}\left(\vec{r}, \int \mathrm{d} \overrightarrow{r^{\prime}} \rho_{A}\left(\overrightarrow{r^{\prime}}\right)\right)}\right) \mathrm{d} \vec{r} .
$$

Since the constraint is linear in the $\rho_{A}(\vec{r})$ variables, we concentrate on the con- 
vexity of the functional in Eq. 26 by calculating the Hessian,

$$
\begin{array}{r}
\frac{\delta^{2} J}{\delta \rho_{B}\left(\overrightarrow{r_{1}}\right) \delta \rho_{C}\left(\overrightarrow{r_{2}}\right)}=\delta_{B C}\left\{\frac{\delta\left(\overrightarrow{r_{1}}-\overrightarrow{r_{2}}\right)}{\rho_{B}\left(\overrightarrow{r_{1}}\right)}-\frac{\Delta \rho_{B}^{0}\left(\overrightarrow{r_{1}}, N_{B}^{0}\left[\rho_{B}\right]\right)}{\rho_{B}^{0}\left(\overrightarrow{r_{1}}, N_{B}^{0}\left[\rho_{B}\right]\right)}-\frac{\Delta \rho_{B}^{0}\left(\overrightarrow{r_{2}}, N_{B}^{0}\left[\rho_{B}\right]\right)}{\rho_{B}^{0}\left(\overrightarrow{r_{2}}, N_{B}^{0}\left[\rho_{B}\right]\right)}\right. \\
\left.+\int \mathrm{d} \vec{r} \rho_{B}(\vec{r})\left(\frac{\Delta \rho_{B}^{0}\left(\vec{r}, N_{B}^{0}\left[\rho_{B}\right]\right)}{\rho_{B}^{0}\left(\vec{r}, N_{B}^{0}\left[\rho_{B}\right]\right)}\right)^{2}-\int \mathrm{d} \vec{r} \frac{\rho_{B}(\vec{r})}{\rho_{B}^{0}\left(\vec{r}, N_{B}^{0}\left[\rho_{B}\right]\right)} \frac{\partial}{\partial N_{B}^{0}}\left(\Delta \rho_{B}^{0}\left(\vec{r}, N_{B}^{0}\left[\rho_{B}\right]\right)\right)\right\},
\end{array}
$$

where

$$
\begin{gathered}
J=\sum_{A} \int \rho_{A}(\vec{r}) \ln \left(\frac{\rho_{A}(\vec{r})}{\rho_{A}^{0}\left(\vec{r}, \int \mathrm{d} \overrightarrow{r^{\prime}} \rho_{A}\left(\overrightarrow{r^{\prime}}\right)\right)}\right) \mathrm{d} \vec{r}, \\
N_{B}^{0}\left[\rho_{B}\right]=\int \mathrm{d} \vec{r} \rho_{B}(\vec{r}),
\end{gathered}
$$

and $\Delta \rho_{A}^{0}$ as defined in Eq. 17 .

We now check the positivity of the Hessian by considering the sign of

$$
\int \mathrm{d} \overrightarrow{r_{1}} \mathrm{~d} \overrightarrow{r_{2}} \psi\left(\overrightarrow{r_{1}}\right) \psi\left(\overrightarrow{r_{2}}\right) \frac{\delta^{2} J}{\delta \rho_{B}\left(\overrightarrow{r_{1}}\right) \delta \rho_{C}\left(\overrightarrow{r_{2}}\right)},
$$

where $\psi(\vec{r})$ is an arbitrary (real) test function. The contribution to Eq. 30 of all but the last term in Eq. 27 is positive, as it can be rewritten as

$$
\int \mathrm{d} \vec{r} \rho_{B}(\vec{r})\left[\frac{\psi(\vec{r})}{\rho_{B}(\vec{r})}-C \frac{\Delta \rho_{B}^{0}\left(\vec{r}, N_{B}^{0}\left[\rho_{B}\right]\right)}{\rho_{B}^{0}\left(\vec{r}, N_{B}^{0}\left[\rho_{B}\right]\right)}\right]^{2},
$$

where

$$
C=\int \mathrm{d} \vec{r} \psi(\vec{r}) .
$$

The last term in Eq. 27 spoils the picture, however. It contains a second derivative of $\rho_{B}^{0}\left(\vec{r}, N_{B}^{0}\right)$ with respect to particle number,

$$
\frac{\partial}{\partial N_{B}^{0}} \Delta \rho_{B}^{0}\left(\vec{r}, N_{B}^{0}\right)=\frac{\partial^{2}}{\partial N_{B}^{0}{ }^{2}} \rho_{B}^{0}\left(\vec{r}, N_{B}^{0}\right),
$$

which is zero almost everywhere, but undefined when $N_{B}^{0}\left[\rho_{B}\right]$ crosses an integer value. So the Hirshfeld-I $\lambda$ functional is only patchwise convex, and general conclusions cannot be drawn. As found from numerical tests, one has the same independence from the initial atomic populations as in Hirshfeld-I.

\section{Computational Details}

The Hirshfeld-I $\lambda$ scheme was implemented using Eqs. 4,19,20,21 and 24. Numerical integrations were performed as proposed by Becke [35]. All molecular densities were calculated with the Gaussian [36] program, at the RHF level of theory, and either in the $6-311++\mathrm{G}^{* *}$ or $3-21 \mathrm{G}$ basis set. The atomic densities 
(for the integer populations $Z_{A}-2$ up to $Z_{A}+2$ ) were calculated with Gaussian, at the ROHF level of theory, and spherically averaged. Molecular and atomic densities were always in the same basis set. Atoms were considered in the electronic ground state. All calculations were performed using molecules out of the previously used test set [34], and all correlation figures and data were made using all molecules out of this test set.

The program started with a Hirshfeld-I calculation, and these HirshfeldI populations were used as the promolecular populations in the first loop of the Hirshfeld-I $\lambda$ scheme. This way the Hirshfeld, Hirshfeld-I and Hirshfeld-I $\lambda$ charges are all computed in a single program run. The order $k$ of the fitting function started at a value of 10, and was raised by 2 in each iteration of the outer loop. The quench factor was chosen as to 0.1. The convergence criterion for the outer loop was $a b s\left(N_{A}^{i}-N_{A}^{i-1}\right)<0.0001$ and $a b s\left(\lambda_{A}^{i}-\lambda_{A}^{i-1}\right)<0.0001$ for all atoms $A$. The convergence criterion for the inner loop was less tight: $\operatorname{abs}\left(N_{A}^{i}-N_{A}^{i-1}\right)<0.001$ and $a b s\left(\lambda_{A}^{i}-\lambda_{A}^{i-1}\right)<0.001$ for all atoms $A$. This poses no threat as the outer loop controls the precision of the end result. If more than 200 iterations were needed in the inner loop before convergence was concluded, the program was considered not able to reach convergence and the calculations were stopped. The populations of the previous $k$-value were then considered as the best possible Hirshfeld- $\mathrm{I} \lambda$ populations.

\section{$5 \quad$ Results and Discussion}

All molecules in the test set [34] were subjected to the Hirshfeld-I $\lambda$ scheme, both in the $3-21 \mathrm{G}$ and $6-311++\mathrm{G}^{* *}$ basis set, at $\mathrm{HF}$ level of theory. All calculations converged with a $k$ value of at least 50, recovering large part of the discontinuity of the Fukui function.

All molecules in the test set were subjected to the Hirshfeld-I $\lambda$ scheme several times, starting from different promolecular populations. Upon convergence, no molecule showed a significant difference between the different runs, reminiscent of earlier findings for Hirshfeld-I [9] and the Iterated Stockholder Atoms method $[15,16]$.

As the AIM is not an observable, AIM charges can not be strictly described as right or wrong and one can merely assess whether they have interpretive power. The fact that all charges are in line with chemical intuition is a first indication that the charges are useful. Some further assessment of their values can be made through comparison with other AIM schemes. In particular, graphs $\mathrm{A}$ and $\mathrm{B}$ of Figure 2 represent the correlation between the Hirshfeld-I $\lambda \mathrm{HF} / 3$ $21 \mathrm{G}$ charges and respectively the Hirshfeld charges and the Hirshfeld-I charges at that same level of theory. Graphs $\mathrm{C}$ and $\mathrm{D}$ show similar correlations but now for $\mathrm{HF} / 6-311++\mathrm{G}^{* *}$ calculations. Multiple conclusions can be drawn from these plots.

Almost all $\mathrm{HF} / 3-21 \mathrm{G}$ Hirshfeld-I $\lambda$ charges are within the interval $[-1.0,1.0]$ with only a few charges in the $3-21 \mathrm{G}$ basis set exceeding +1.0 . These highly positive charges are found for carbon atoms that have several electronegative neighboring atoms. In comparison: the Hirshfeld-I charges lie in the interval $[-1.0,1.5]$ with some exceptions, while almost all Hirshfeld charges lie in the interval $[-0.4,0.4]$

Table I summarizes the regression equations and correlation coefficients of 

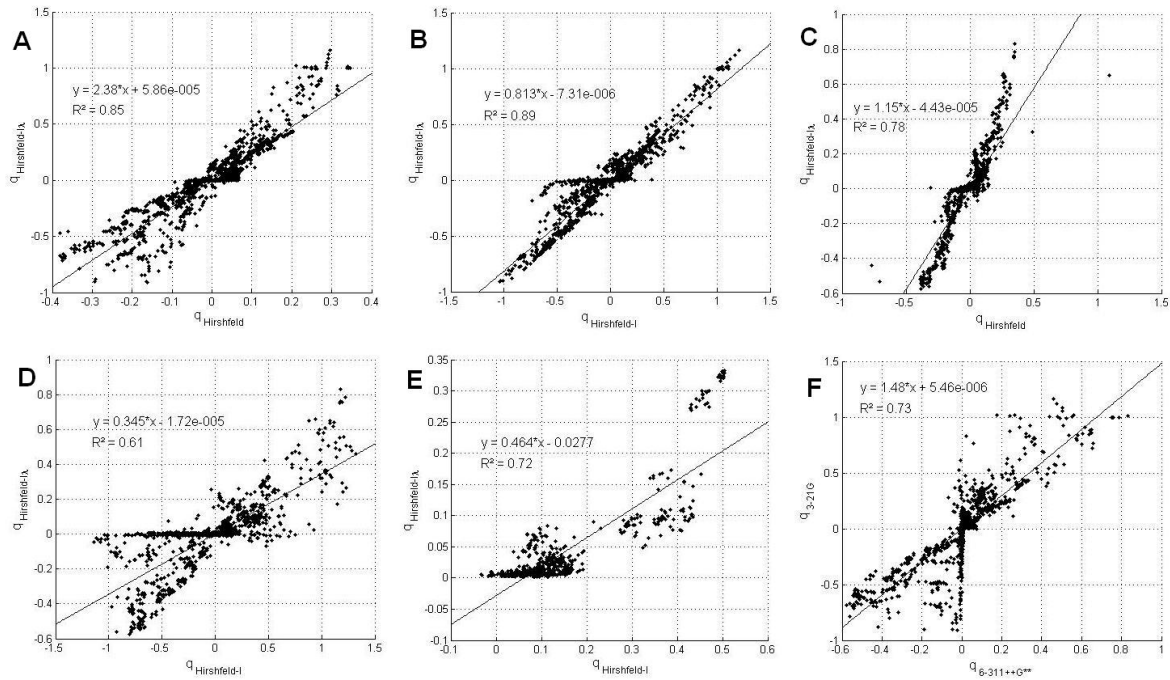

Figure 2: Correlation plots for Hirshfeld-I $\lambda$ charges.

A. Hirshfeld-I $\lambda$ charges vs. Hirshfeld charges (HF/3-21G).

B. Hirshfeld-I $\lambda$ charges vs. Hirshfeld-I charges (HF/3-21G).

C. Hirshfeld-I $\lambda$ charges vs. Hirshfeld charges (HF/6-311++ $\left.\mathrm{G}^{* *}\right)$.

D. Hirshfeld-I $\lambda$ charges vs. Hirshfeld-I charges (HF/6-311++G**).

E. Hirshfeld-I $\lambda$ charges vs. Hirshfeld-I charges on hydrogen atoms

$\left(\mathrm{HF} / 6-311++\mathrm{G}^{* *}\right)$.

F. Hirshfeld-I $\lambda$ charges in the $3-21 \mathrm{G}$ basis set vs. the $6-311++\mathrm{G}^{* *}$ basis set (HF). 


\begin{tabular}{ccc}
\hline $\mathrm{HF} / 3-21 \mathrm{G}$ & $q_{\mathrm{HI \lambda}}=2.38 q_{\mathrm{H}}$ & $R^{2}=0.85$ \\
& $q_{\mathrm{HI} \lambda}=0.813 q_{\mathrm{HI}}$ & $R^{2}=0.89$ \\
\hline $\mathrm{HF} / 6-311++\mathrm{G}^{* *}$ & $q_{\mathrm{HI \lambda}}=1.15 q_{\mathrm{H}}$ & $R^{2}=0.78$ \\
& $q_{\mathrm{HL} \lambda}=0.345 q_{\mathrm{HI}}$ & $R^{2}=0.61$ \\
\hline
\end{tabular}

Table I: Regression equations and correlation coefficients between Hirshfeld-I $\lambda$ charges and Hirshfeld or Hirshfeld-I charges.

\begin{tabular}{ccc}
\hline Hirshfeld & $y=1.0 x$ & $R^{2}=0.99$ \\
Hirshfeld-I & $y=1.1 x$ & $R^{2}=0.99$ \\
Hirshfeld-I $\lambda$ & $y=1.48 x$ & $R^{2}=0.73$ \\
\hline
\end{tabular}

Table II: Regression equations and correlation coefficients between charges in the $3-21 \mathrm{G}$ basis set and the $6-311++\mathrm{G}^{* *}$ basis set, at HF level of theory.

the plots A to D in Figure 2. The equations show that the Hirshfeld-I $\lambda$ charges in both basis sets lie in between the Hirshfeld and the Hirshfeld-I charges. The charges in the 3-21G basis set are closer to the Hirshfeld-I charges, with a relatively good correlation between both methods. At the $\mathrm{HF} / 6-311++\mathrm{G}^{* *}$ level, the correlation with the Hirshfeld-I charges is much worse, and the charges are closer to the Hirshfeld charges, so no general conclusion can be drawn.

Graph E of Figure 2 reveals that $\mathrm{HF} / 6-311++\mathrm{G}^{* *}$ hydrogen charges are very small. They even can grow smaller than the Hirshfeld charges, which are significantly smaller already than the Hirshfeld-I charges [9]. Only those hydrogen atoms attached to an electronegative atom (such as oxygen or nitrogen) have a significantly positive charge. The charges on the carbon atoms show the same trend as the hydrogen atoms. The same is true at the HF/3-21G level although there the effect is smaller as is clear from plot B in Figure 2.

There seems to be a large basis-set dependence, as the Hirshfeld- $\mathrm{I} \lambda$ charges in the different basis sets differ substantially. The Hirshfeld-I $\lambda$ charges in the 3-21G basis set are more pronounced than the charges in the $6-311++\mathrm{G}^{* *}$ basis set. Plots A to D of Figure 2 show that most HF $/ 6-311++\mathrm{G}^{* *}$ Hirshfeld-I $\lambda$ charges lie in the interval $[-0.6,0.6]$; in contrast to the charges in the $3-21 \mathrm{G}$ basis set, largely confined to the interval $[-1.0,1.0]$. This basis-set dependence is quantified in graph F of Figure 2, and Table II, and is also visible in Table I. Table II shows that the Hirshfeld and Hirshfeld-I charges are practically basis-set independent. The Hirshfeld-I $\lambda$ charges on the other hand differ substantially: the average charge in the $3-21 \mathrm{G}$ basis set is approximately one and a half times the average charge in the $6-311++\mathrm{G}^{* *}$ basis set.

Finally, the electrostatic potentials calculated with the Hirshfeld-I $\lambda$ charges correlate unfavorably with ab initio electrostatic potentials computed on a grid of points on and near the molecular Van der Waals surface (see [24] for technical details). Potentials computed with a point charge model using Hirshfeld and particularly Hirshfeld-I charges correlate much better [24], as is illustrated in Table III. 


\begin{tabular}{ccc}
\hline & $R M S E$ & $R^{2}$ \\
\hline Hirshfeld & 0.0087 & 0.80 \\
Hirshfeld-I & 0.0047 & 0.86 \\
Hirshfeld-I $\lambda$ & 0.0079 & 0.74 \\
\hline
\end{tabular}

Table III: Root mean squared errors (RMSE) and correlation coefficients between electrostatic potentials calculated with the charge method used and ab initio electrostatic potentials on a series of Van der Waals surfaces (at HF/3-21G level of theory).

\section{Conclusion}

A new iterative Hirshfeld type AIM method, called Hirshfeld-I $\lambda$, has been proposed. The weight function that defines these AIM is constructed by minimizing the information loss on formation of the molecule, explicitly requiring that the promolecular densities integrate to the same number of electrons as the AIM densities constructed. The atoms defined by this AIM method are the ones that minimize the information lost upon formation of the molecule out of its isolated atoms. The new AIM method was implemented and the results were examined. The Hirshfeld-I $\lambda$ charges are unique, and are only defined by the atomic and molecular densities. There is no theoretical proof, but this statement is backed by results gathered through extensive testing. All charges are in line with chemical intuition. The Hirshfeld-I $\lambda$ charges are in general larger than the Hirshfeld charges and smaller than the Hirshfeld-I charges. The more electronegative elements have charges closer to those of the Hirshfeld-I scheme. In contrast to the Hirshfeld and Hirshfeld-I charges, the Hirshfeld-I $\lambda$ charges show great basis-set sensitivity. The charges in the $3-21 \mathrm{G}$ basis set are on average one and a half times the charges in the $6-311++\mathrm{G}^{* *}$ basis set. As a result, the charges in the $3-21 \mathrm{G}$ basis set lie closer to the Hirshfeld-I charges, while the charges in the larger basis set lie closer to the Hirshfeld charges. Another point of criticism on the Hirshfeld-I $\lambda$ scheme originates from the electrostatic potentials calculated with these charges. The calculated electrostatic potentials correlate badly with ab initio electrostatic potentials, while the Hirshfeld and especially the Hirshfeld-I scheme perform much better. The Hirshfeld-I scheme is therefore considered the best of the three schemes.

\section{$7 \quad$ Acknowledgments}

We gratefully acknowledge financial support from the FWO-Flanders and the research council of Ghent University. P.W.A. acknowledges support from NSERC and Sharcnet. 
[1] Mulliken, R. S. J Chem Phys 1955, 23, 1833.

[2] Mulliken, R. S. J Chem Phys 1955, 23, 1841.

[3] Mulliken, R. S. J Chem Phys 1955, 23, 2338.

[4] Mulliken, R. S. J Chem Phys 1955, 23, 2343.

[5] Chattaraj, P. K. Chemical reactivity theory: a density functional view; CRC Press Taylor and Francis Group: Boca Raton, 2009; Chapter 15.

[6] Bader, R. F. W. Chem Rev (Washington D.C.) 1991, 91, 893.

[7] Bader, R. F. W. Atoms in molecules: a quantum theory; Clarendon: Oxford, 1990.

[8] Hirshfeld, F. L. Theoret Chim Acta (Berl.) 1977, 44, 129-138.

[9] Bultinck, P.; Van Alsenoy, C.; Ayers, P. W.; Carb-Dorca, R. J Chem Phys 2007, 126, 144111.

[10] Davidson, E. R.; Chakravorty, S. Theor Chim Acta 1992, 83, 319-330.

[11] Bader, R. F. W.; Matta, C. F. J Phys Chem A 2004, 108, 8385-8394.

[12] Matta, C. F.; Bader, R. F. W. J Phys Chem A 2006, 110, 6365-6371.

[13] Bultinck, P. Faraday Discuss 2007, 135, 244-246.

[14] Lillestolen, T. C.; Wheatley, R. J. Chem Commun 2008, 45, 5909-5911.

[15] Lillestolen, T. C.; Wheatley, R. J. J Chem Phys 2009, 131, 144101.

[16] Bultinck, P.; Cooper, D. L.; Van Neck, D. Phys Chem Chem Phys 2009, $11,3424-3429$.

[17] Perdew, J. P.; Parr, R. G.; Levy, M.; Balduz Jr, J. L. Phys Rev Lett 1982, 49, 1691.

[18] Yang, W.; Zhang, Y.; Ayers, P. W. Phys Rev Lett 2000, 84, 5172-5175.

[19] Ayers, P. W. J Math Chem 2008, 43, 285-303.

[20] Bultinck, P.; Fias, S.; Van Alsenoy, C.; Ayers, P. W.; Carb-Dorca, R. J Chem Phys 2007, 127, 034102.

[21] Ayers, P. W.; Levy, M. Theor Chem Acc 2000, 103, 353-360.

[22] Ayers, P. W.; De Proft, F.; Borgoo, A.; Geerlings, P. J Chem Phys 2007, 126, 224107. 
[23] Bultinck, P.; Ayers, P. W.; Fias, S.; Tiels, K.; Van Alsenoy, C. Chem Phys Lett 2007, 444, 205-208.

[24] Van Damme, S.; Bultinck, P.; Fias, S. J Chem Theory Comput 2009, $5(2), 334-340$.

[25] Nalewajski, R. F.; Parr, R. G. Proc Natl Acad Sci U.S.A. 2000, 97, 8879-8882.

[26] Nalewajski, R. F.; Switka, E.; Michalak, A. Int J Quantum Chem 2002, $87,198-213$.

[27] Nalewajski, R. F. Chem Phys Lett 2003, 372, 28-34.

[28] Nalewajski, R. F.; Broniatowska, E. Int J Quantum Chem 2005, 101, 349-362.

[29] Parr, R. G.; Ayers, P. W.; Nalewajski, R. F. J Phys Chem A 2005, 109, 3957-3959.

[30] Pierce, J. R. An introduction to information theory: symbols, signals and noise; Dover: New York, 1980.

[31] Kullback, S. Information theory and statistics; Wiley: New York, 1959.

[32] Parr, R. G.; Yang, W. J Am Chem Soc 1984, 106, 4049-4050.

[33] Yang, W.; Parr, R. G.; Pucci, R. J Chem Phys 1984, 81, 2862-2863.

[34] The set with the testing molecules can be found via the following link http://ftp.aip.org/epaps/journ_chem_phys/E-JCPSA6-126-309713/supplementary.pdf or via the EPAPS homepage: http://www.aip.org/pubservs/epaps.htm, with document number E-JCPSA6-126-309713.

[35] Becke, A. D. J Chem Phys 1988, 88(4), 2547-2553.

[36] Frisch, M. J. ; Trucks, G. W.; Schlegel, H. B.; Scuseria, G. E.; Robb, M. A.; Cheeseman, J. R.; Montgomery, Jr. J. A.; Vreven, T.; Kudin, K. N.; Burant, J. C.; Millam, J. M.; Iyengar, S. S.; Tomasi, J.; Barone, V.;Mennucci, B. ;Cossi, M. ;Scalmani, G. Rega,; N.; Petersson, G. A.; Nakatsuji, H.; Hada, M.; Ehara, M.; Toyota, K. ; Fukuda, R.; Hasegawa, J.; Ishida, M.; Nakajima, T.; Honda, Y.; Kitao, O.; Nakai, H.; Klene, M.; Li,X.; Knox, J. E.; Hratchian, H. P.; Cross, J. B.; Bakken, V.; Adamo, C.; Jaramillo, J.; Gomperts, R.; Stratmann, 
R. E.; Yazyev, O.; Austin, A. J.; Cammi, R.; Pomelli, C.; Ochterski, J. W.; Ayala, P. Y.; Morokuma, K.; Voth, G. A.;Salvador, P. ; Dannenberg, J. J.; Zakrzewski, V. G.; Dapprich, S.; Daniels, A. D.; Strain, M. C.; Farkas, O.; Malick, D. K.; Rabuck, A. D.; Raghavachari, K.; Foresman, J. B.; Ortiz, J. V.; Cui, Q.; Baboul, A. G.; Clifford, S.; Cioslowski, J.; Stefanov, B. B.; Liu, G.; Liashenko, A.; Piskorz, P.; Komaromi, I.; Martin, R. L.; Fox, D. J.; Keith, T.; Al-Laham, M. A.; Peng, C. Y.; Nanayakkara, A.; Challacombe, M.; Gill, P. M. W.; Johnson, B.; Chen, W.; Wong, M. W.;Gonzalez, C. ; Pople, J. A. Gaussian 03 Revision E.01 2004; Gaussian Inc., Wallingford CT. 